\title{
A CASE OF OSTEOSARCOMA OF THE MAXILLA - CHALLENGES IN DIAGNOSIS AND MANAGEMENT
}

N.I.H. Abdul Rahim, N.A. Ngah, A. Ramanathan, T. George, S.M. Ismail. A case of osteosarcoma of the maxilla - challenges in diagnosis and management. Annal Dent Univ Malaya 2011; 18: 24-29.

\begin{abstract}
Osteosarcoma is a primary malignant neoplasm of the bone. Osteosarcoma of the jaws especially those of maxilla is rare. The diagnosis of osteosarcomas is difficult and challenging. In this case report we highlight a rare case of osteosarcoma of the maxilla in a 29 year old male patient which was highly aggressive and was initially diagnosed as rhabdomyosarcoma. This case highlights the difficulty in diagnosing osteosarcoma merely from incisional biopsy specimens which may not be representative of the whole tumour. Limited clinical information at incisional biopsy also adds to the difficulty in arriving at the definitive diagnosis. We further discuss the treatment modalities followed in this case.
\end{abstract}

Key words: Osteosarcoma, jaws, maxilla, diagnosis, treatment

\section{INTRODUCTION}

Osteosarcomas are a heterogeneous group of malignant tumours affecting the bone or mesenchymal tissues characterized by formation of disorganized woven bone or osteoid tissue (1). The aetiology of osteosarcomas is still unknown but most of them contain clonal chromosomal aberrations (2). Osteosarcomas are the second most common primary bone tumours after multiple myeloma and accounts for $15-35 \%$ of all primary bone tumours $(3,4)$. The estimated incidence of osteosarcomas is $4-5$ cases per million population (4).

Osteosarcomas occur most commonly in the long bones but are rare in the jaws with an estimated incidence of 0.7 cases per million. Osteosarcomas of the jaw have a slight male predilection (5). Patients with long bone lesions tend to be in their 2 nd to $3 \mathrm{rd}$ decades whereas; patients with osteosarcoma of the jaws tend to be in their 4th to 5th decades of life (6). Osteosarcomas occur more frequently in the mandible than in the maxilla (7). The posterior body and the horizontal ramus are commonly involved regions in the mandible, whereas in the maxilla, the alveolar ridge, sinus floor and palate are the most commonly involved (8). Paget's disease, fibrous dysplasia and
Case Report

N.I.H. Abdul Rahim ${ }^{1}$, N.A. Ngah ${ }^{2}$,
A. Ramanathan ${ }^{3,4}, \mathrm{~T}$. George ${ }^{3,4}$, S.M. Ismail ${ }^{4,5}$
${ }^{1}$ Department of Oral \& Maxillofacial Surgery
Hospital Kuala Lumpur
Kuala Lumpur, Malaysia.
${ }^{2}$ Centre of Studies for Oral \& Maxillofacial Surgery
Faculty of Dentistry, Universiti Teknologi MARA,
Kuala Lumpur, Malaysia.
${ }^{3}$ Department of Oral Pathology Oral Medicine \&
Periodontology
${ }^{4}$ Oral Cancer Research and Coordinating Centre
(OCRCC)
${ }^{5}$ Department of Oral \& Maxillofacial Surgery
Faculty of Dentistry, University of Malaya,
50603, Kuala Lumpur, Malaysia.
Tel. No. (Office): 03-79674801
Email: sitimazlipah@um.edu.my
Corresponding author: Dr. Siti Mazlipah Ismail

previous radiation therapy are known predisposing factors of osteosarcoma $(3,9)$.

The presenting symptom depends on the location of the tumour and may be vague or non-exixtent (10). Pain, swelling, paraesthesia and pathological fracture may occur later in the disease process (10). Intratumoural haemorrhage, infection or dedifferentiation may cause sudden and dramatic increase in the size of the lesion (10). Histologically, osteosarcomas are subdivided into chondroblastic, osteoblastic and fibroblastic variants (8). They may also be graded as high, intermediate and low grade osteosarcomas. Based on location they may be subdivided into central (medullary) and peripheral (surface) types. The peripheral osteosarcoma may be either parosteal (juxtacortical) or periosteal in location (11).

These tumours have an ability to metastasize via blood stream and are considered as a prototype mesenchymal malignancy (12). However, osteosarcomas of the jaw have a low incidence of metastasis and better prognosis (13).

In this case report we highlight a rare case of osteosarcoma of the maxilla in a 29 year old male patient which was highly aggressive and posed a diagnostic dilemma at the initial histopathological examination. We further discuss the treatment modalities followed in this case. 


\section{CASE REPORT}

A 29 year old male patient, presented to the Oral and Maxillofacial Surgery Clinics in our institution on December 2007 with a rapidly expanding, painful mass on his palate of 4 weeks duration. He had complained of toothache in his left upper molars prior to the development of the swelling and was prescribed analgesics and antibiotics by a private dental practitioner. However the swelling persisted, hence the patient was referred to another Oral and Maxillofacial centre, where an incisional biopsy was done. Following the biopsy the swelling exhibited rapid expansion and the patient was referred to our centre.

The past medical history of the patient was noncontributory. However, he was a smoker and had no other habits. He had no loss of appetite but observed a sudden loss of weight in the recent period. On intraoral examination, there was a growth approximately $5 \mathrm{~cm} \times 3 \mathrm{~cm} \times 2 \mathrm{~cm}$ in size on the hard palate. The pedunculated growth extended to the softpalate and uvula. It was irregular in shape, lobulated and the surface was covered with necrotic slough (Figure 1). Teeth 24 to 26 had grade II mobility. Patient had difficulty in speaking and eating.

The CT scan showed an ill-defined, heterogenous, enhancing soft tissue mass measuring $6.4 \mathrm{~cm} \mathrm{x} 3.0 \mathrm{~cm}$ $\mathrm{x} 2.5 \mathrm{~cm}$ arising from the hard palate (Figure 2). The medial wall of the left maxillary sinus showed destruction. Several cervical lymph nodes were enlarged with the largest being a right posterior cervical lymph node measuring $1.5 \mathrm{~cm}$ in diameter.

Incisional biopsy findings performed at another centre prior to the patient being referred to our centre confirmed a diagnosis of rhabdomyosarcoma as the tumour cells were immune-positive for vimentin, desmin and moderately positive for actin. The patient was administered chemotherapy due to the extensiveness of the tumour. The patient received adriamycin and ifosfamide. Initially, the tumour showed a positive response as evidence by reduction in size however, a week after the induction of chemotherapy, the tumour started to rapidly increase in size causing severe pain and discomfort to the patient (Figure 3). An emergency surgery to debulk the tumour was done to prevent airway obstruction. Two weeks later, a left subtotal maxillectomy was carried out.

The histopathology of the surgical specimen revealed, surface parakeratinized stratified squamous epithelium and the underlying connective tissue densely infiltrated with tumour cells. The neoplastic cells were highly pleomorphic, and round to polygonal in shape. Few areas showed spindle cells (Figure 4A). The neoplastic cells showed enlarged hyperchromatic nuclei and inconspicuous cytoplasm against a background of hyalinised and myxoid stroma. Numerous mitotic figures were present. Areas of osteoid formation (Figure 4B) and chondroid differentiation (Figure 4C) were evident. The neoplastic cells appeared to invade adjacent lobules of minor salivary glands. Dilated blood vessels with evidence of perivascular and intravascular invasion of tumour cells were evident. Perineural invasion was also observed (Figure 4D). The tumour cells stained negatively with myogenin. Hence a definitive diagnosis of osteoblastic ostoesarcoma was given.

The post-operative recovery of the patient was uneventful (Figure 5). He later underwent adjuvant chemotherapy with doxorubicin and cisplatin. At review prior to cycle 3 he was well with no evidence of disease recurrence. Unfortunately after completion of cycle 3 , the patient defaulted treatment and followup.

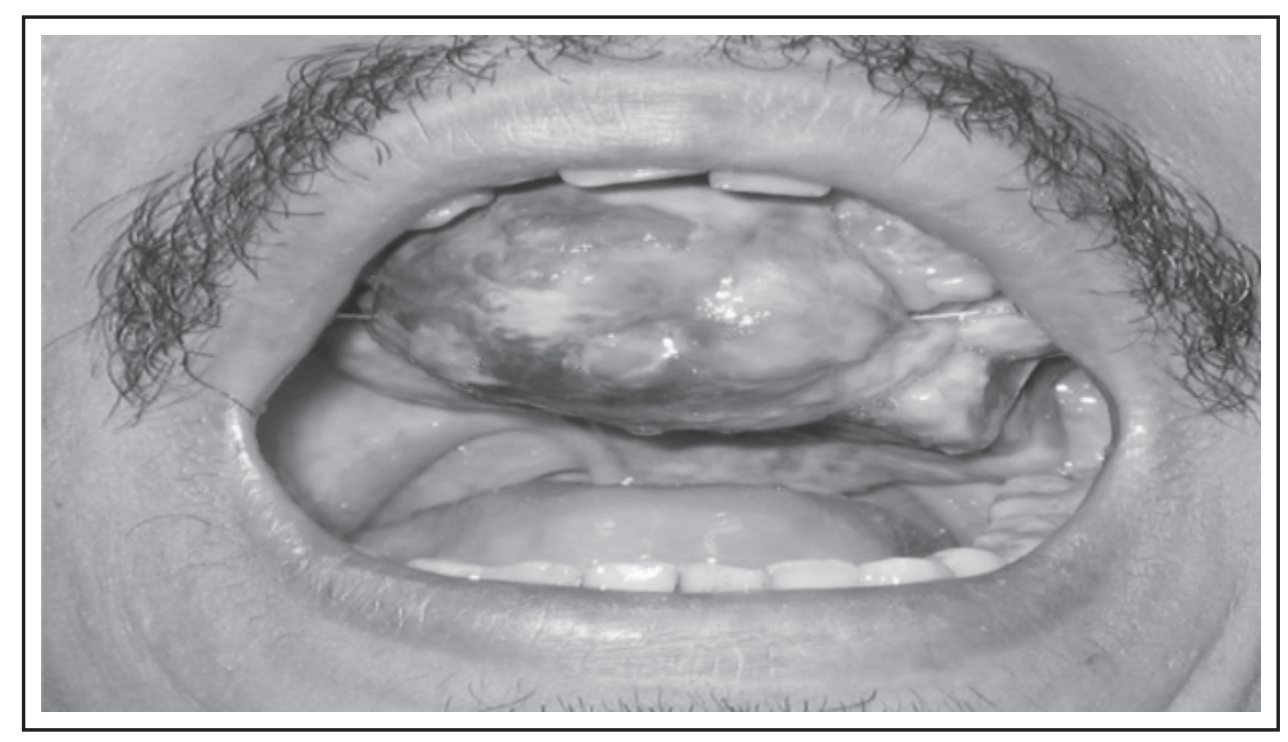

Figure 1. An intra-oral photograph showing a growth occupying the whole of hard palate and extending to the soft palate and uvula. 


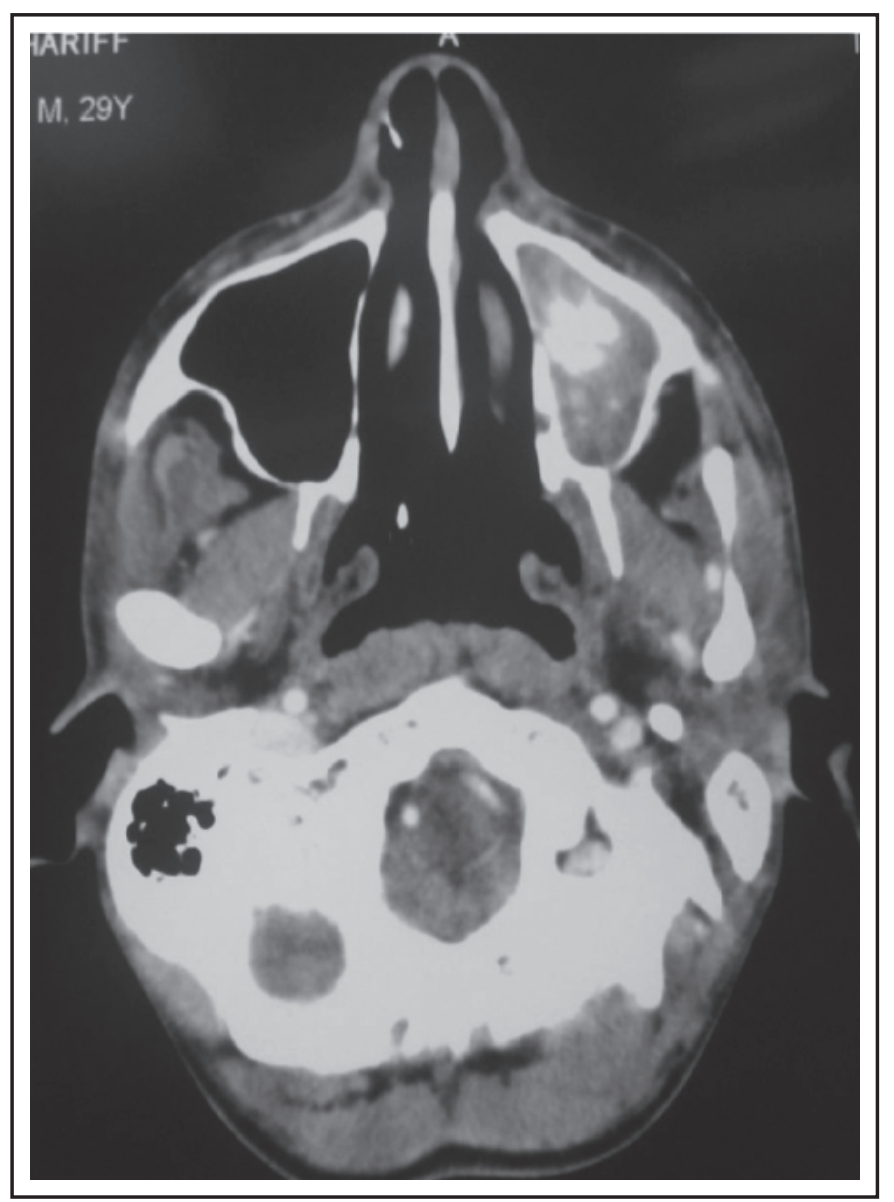

Figure 2. Preoperative axial CT scan showing the tumour mass in the left maxilla.

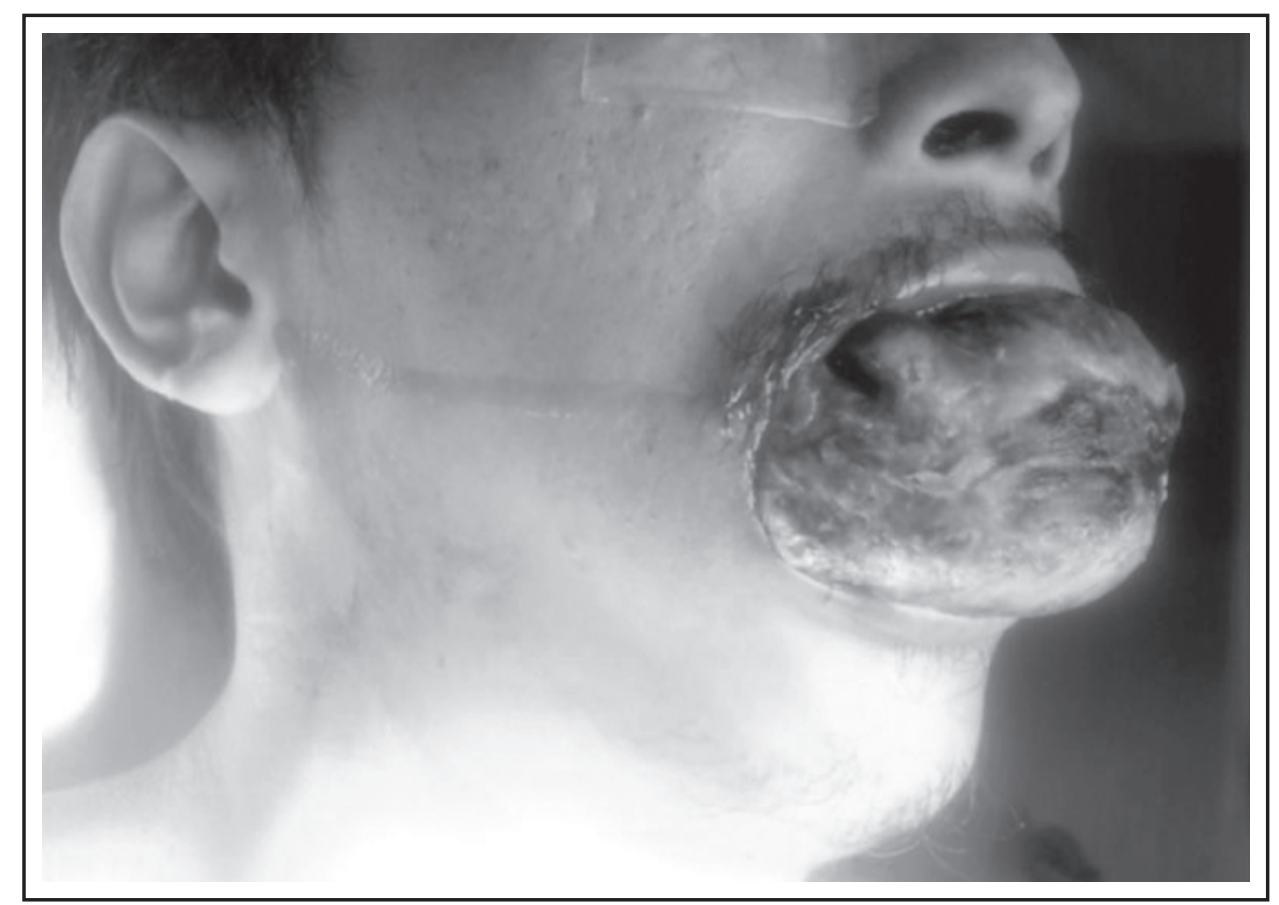

Figure 3. Photograph showing the aggressively expanded tumour after a week of chemotherapy. 


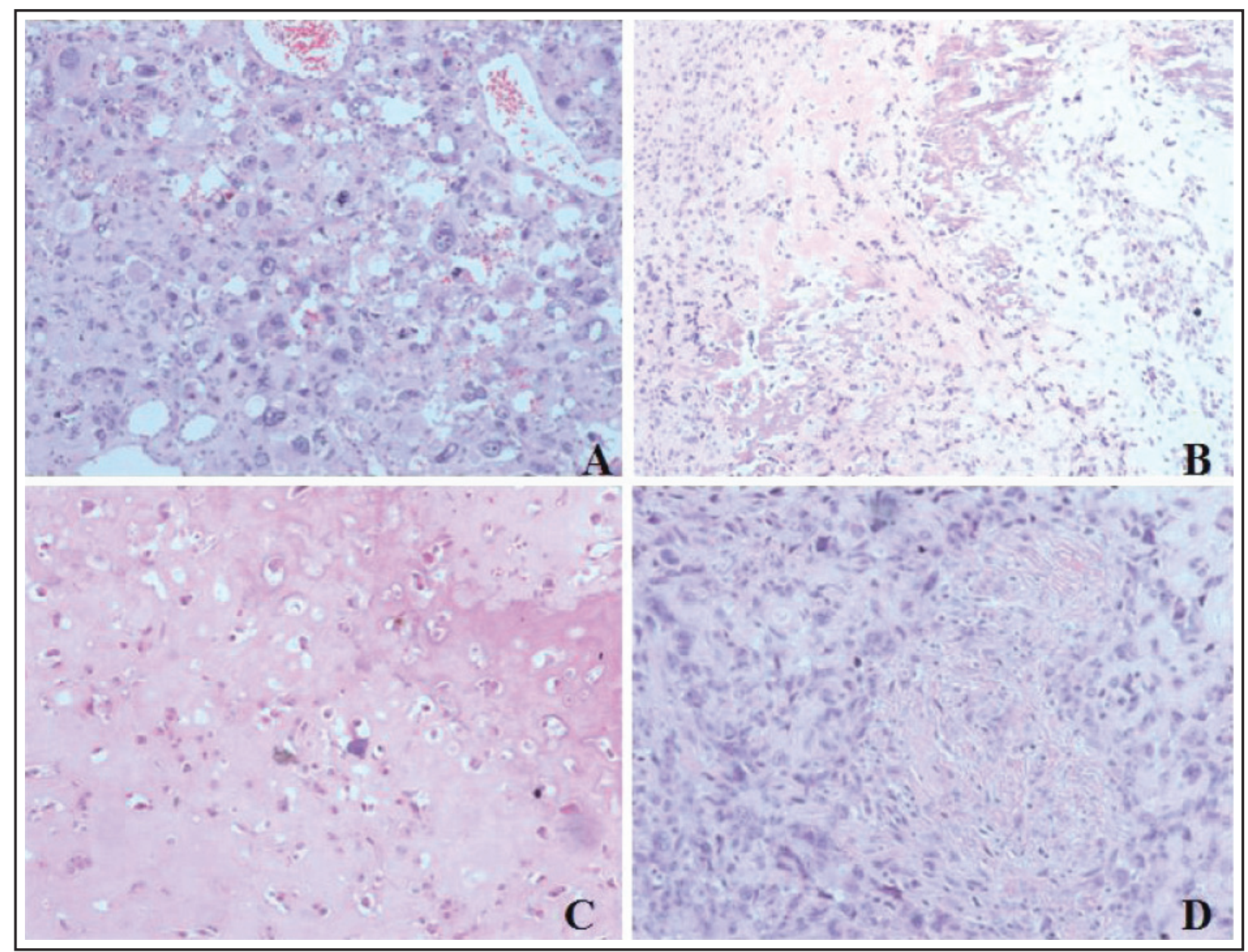

Figure 4. (A): Photomicrograph showing the connective tissue infiltrated with highly pleomorphic round to polygonal neoplastic cells (Original Magnification 20x). (B): Areas showing osteoid formation (Original Magnification 10x). (C): Photomicrograph showing chondroid differentiation (Original Magnification 40x). (D): Photomicrograph showing perineural invasion of neoplastic cells (Original Magnification 20x).

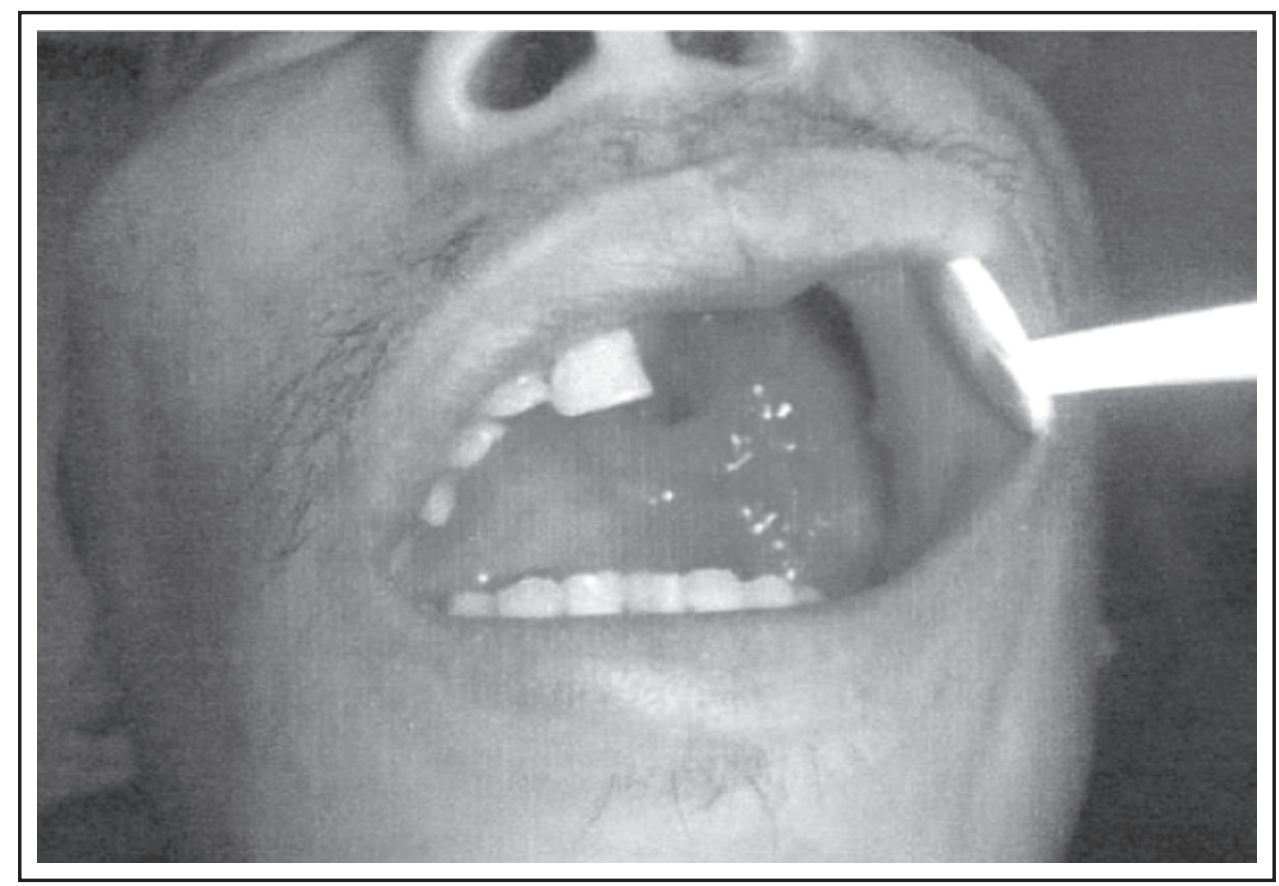

Figure 5. Photograph of the postoperative intra-oral view. 


\section{DISCUSSION}

Osteosarcomas are primary malignant bone tumours (3) and majority of them arise de novo, whereas some develop in association with conditions such as Paget's disease, fibrous dysplasia, chronic osteomyelitis, trauma or previous radiation therapy $(3,9,14)$. Maxillary osteosarcomas are rare (7) with a peak incidence in the 4th to 5th decades (6), which is 10 to 15 years later than the mean age osteosarcomas occurring within the long bones $(8,14,15)$. However, the age range at presentation for jaw lesions varies widely between 7 and 63 years (10). We present a rare case of osteosarcoma of the maxilla which developed de novo in a male patient who was in his 3rd decade.

The clinical diagnosis of osteosarcoma is difficult as the clinical signs and symptoms such as swelling, pain and general discomfort are non-specific findings $(4,8,14)$. Symptoms of osteosarcoma of the jaws may be absent or minimally apparent during the early disease state but eventually progresses to an expansible mass with neurosensory changes such as paresthesia (10). Displacement and mobility of teeth may also be evident (8). Our patient presented with a rapidly expanding painful mass on the palate associated with tenderness over the left upper molar teeth.

The differential diagnosis for an expansile mass of the jaw may include a large number of entities for example ossifying fibroma, giant cell granuloma, odontogenic cysts and tumours, fibrous dysplasia, ossifying fibromas and benign and malignant neoplasms. A tissue biopsy is the only means of making a definitive diagnosis however the incisional biopsy is not without pitfalls. As in our case the initial incisional biopsy was diagnosed as rhabdomyosarcoma by another centre.

Osteosarcomas of the jaws may be misdiagnosed as example fibrous dysplasia, ossifying fibroma (16), osteoma, chondromyxoid fibroma, chondrosarcoma (17), giant cell granuloma (11) and pyogenic granuloma (10). In an extensive lesion, different histopathologic patterns may occur at various sites and therefore an incisional biopsy taken at the surface may not be representative of the true lesion. This is in agreement with Bertoni et al (1991) as they claimed that the histological diagnosis of ostoesarcoma in the jaw is not always straight. Biopsies should be representative of the lesion to increase the accuracy of the pathologic interpretation as small biopsies may not capture the osteoid component (10). In our case, the initial biopsy specimen failed to capture the osteoid component whereas the surgical excision specimens showed the various patterns of appearance of osteosarcoma in different sections which lead to the definitive diagnosis.

Surgery with adequate margins remains the mainstay of treatment for osteosarcomas (19). Osteosarcomas arising from the maxilla cannot always be resected with sufficient margin. This is reflected by a relatively high local recurrence rate (20). Adjuvant chemotherapy has played an important role in increasing the survival of patients with osteosarcoma of the long bones (4). Oda et al (1997) suggested that while pre-operative chemotherapy may not statistically improve survival, it did make the surgical resection easier. However, such reduction in size did not persist as shown in our case. Pre-operative chemotherapy had an initial positive response by way of reduction in size of the lesion that was later followed by an aggressive expansion of the lesion which prompted the surgeons to carry out an emergency de-bulking surgery to prevent airway obstruction.

In the osteosarcoma of the long bones neoadjuvant chemotherapy is carried out before surgery, after which the resected specimen is examined for histological response to the neoadjuvant chemotherapeutic agents and the decision of whether to use the same chemotherapeutic agents or change to other adjuvant chemotherapeutic agents is determined later (22). In our case, neoadjuvant chemotherapy with adriamycin and ifosfamide resulted in an aggressive expansion of the lesion. A definitive diagnosis of osteosarcoma was made later which lead to the change in decision of the choice of cheomotherapeutic agents to doxorubicin and cisplatin for the adjuvant chemotherapy.

\section{CONCLUSION}

Patients with osteosarcoma of the jaws will usually first present to the dental professional due to the clinical presentation of pain and swelling of the jaws. The diagnosis of osteosarcoma of the jaws is often challenging, especially when the initial incisional biopsy specimen is not representative of the true lesion. Therefore, adequate sampling of the lesion should be mandatory to arrive at the definitive diagnosis which can lead to prompt treatment.

\section{ACKNOWLEDGMENT}

We would like to acknowledge Prof. Dr. Rosnah Binti Mohd Zain for her inputs on preparing this article.

\section{REFERENCES}

1. Yesilova E, Akgünlü F, Dolanmaz D, et al. Osteosarcoma: A case report. Eur J Dent. 2007; 1: 60-3.

2. Fletcher CDM, Unni KK, Mertens F, editors. World Health Organization classification of tumors. Pathology and genetics of tumors of soft tissue and bone. Lyon: IARC Press; 2002. 
3. Raymond AK, Ayala AG, Knuutila S. Conventional osteosarcoma. In, Fletcher CD, Unni KK, Mertens F (ed). Pathology and Genetics of Tumors of Soft Tissue and Bone, 1st edition. IARCPress, 2002; 264-270.

4. Fernandes R, Nikitakis NG, Pazoki A, et al. Osteogenic sarcoma of the jaw: A 10-year experience. J Oral Maxillofac Surg. 2007; 65: 1286-91.

5. Barnes L, Eveson JW, Reichart P, et al. editors. World Health Organization classification of tumours. Pathology and genetics of head and neck tumours. Lyon: IARC Press; 2005.

6. Cheng YS, Wright JM, Walstad WR, et al. Osteosarcoma arising in Paget's disease of the mandible. Oral Oncol. 2002; 38(8): 785-92.

7. Bianchi SD, and Boccardi A. Radiological aspects of osteosarcoma of the jaws. Dentomaxillofac Radiol. 1999; 28: 42-7.

8. Angela C. Bone Pathology. In, Neville BW, Damm DD, Allen CM, Bouquot JE, (ed). Oral \& Maxillofacial Pathology, 3rd edition. Saunders, 2009; 660-664.

9. Ogunlewe MO, Ajayi OF, Adeyemo WL, et al. Osteogenic sarcoma of the jaw bones: A single institution experience over a 21-year period. Oral Surg Oral Med Oral Pathol Oral Radiol Endod 2006; 101: 76-81.

10. Padilla RJ, and Murrah VA. The Spectrum of Gnathic Osteosarcoma: Caveats for the Clinician and the Pathologist. Head and Neck Pathol 2011; 5: 92-99.

11. George A, Mani V, Sunil S, et al. Osteosarcoma of Maxilla: A Case of Missed Initial Diagnosis. Oral Maxillofacial Pathology Journal 2010; 1(1).

12. Sheikh S, Pallagatti S, Aggarwal A, et al. Osteosarcoma of maxilla: A case report. J Clin Exp Dent. 2010; 2(3): e117-20.
13. Amaral MB, Buchholz I, Freire-Maia B, et al. Advanced osteosarcoma of the maxilla: A case report. Med Oral Patol Oral Cir Bucal. 2008; 13: E492-5.

14. Rajendran R. Benign and Malignant tumors of the oral cavity. In, Rajendran R, Sivapathasundaram B (ed). Shafer's Textbook of Oral Pathology, 6th edition. Elsevier, 2009; 169-173.

15. Zarbo RJ, and Carlson ER. Malignancies of the Jaws. In, Regezi JA, Sciubba JJ, Jordan RK (ed). Oral Pathology, 5th edition. Saunders, 2008; 315 321 .

16. Bennett JH, Thomas G, Evans AW, et al. Osteosarcoma of the jaws: A 30-year retrospective review. Oral Surg Oral Med Oral Pathol Oral Radiol Endod. 2000; 90(3): 323-32.

17. Baghaie F, and Motahhary P. Osteosarcoma of the jaws: A retrospective study. Acta Medica Iranica. 2003; 41(2): 113-21.

18. Bertoni F, Dallera P, Bacchini $\mathrm{P}$, et al. The instate Rizzoli-Beretta experience with osteosarcoma of the jaw. Cancer 1991; 68 (7): 1555-63.

19. Kassir RR, Rassekh CH, Kinsella JB, et al. Osteosarcoma of the head and neck: Meta-analysis of nonrandomized studies. Laryngoscope. 1997; 107: $56-61$.

20. Russ JE, and Jesse RH. Management of osteosarcoma of the maxilla and mandible. Am J Surg. 1980; 140-72.

21. Oda D, Bavisotto LM, Schmidt RA, et al. Head and neck osteosarcoma at the University of Washington. Head Neck 1997; 19: 513-23.

22. Rosen G, Capparros B, Huvos AG, et al. Preoperative chemotherapy for osteogenic sarcoma: Selection of postoperative adjuvant chemotherapy based on the response of the primary tumor preoperative chemotherapy. Cancer 1982; 49: 1221-30. 